\title{
Sequencing or not sequencing multikinase inhibitors in kidney cancer: this is the dilemma
}

\author{
Chiara Paglino $\cdot$ Camillo Porta
}

Published online: 9 February 2010

(C) Springer-Verlag 2010

With the recent development of targeted therapies (Sorafenib, Sunitinib, Temsirolimus, Bevacizumab plus Interferon- $\alpha$, Everolimus and now also Pazopanib) patients with advanced renal cell carcinoma (RCC) now have a wide range of treatment options, all of which have shown both relevant clinical activity and manageable safety profile. This abundance of active treatments, coupled with relatively limited information, we have gathered from registrative phase III trials have raised the question of how to use these agents optimally.

Indeed, since presently, a cure for advanced RCC is definitely out of sight-despite the improvements made so far-the goal of therapy should be to extend progressionfree survival (PFS) while maintaining a patient's quality of life.

To achieve such a goal, sequencing of novel agents is a therapeutical strategy that is becoming more and more popular in everyday's clinical practice, being mainly supported by the retrospective evaluation of several case series.

Therefore, a recent prospective study reported by $\mathrm{Di}$ Lorenzo et al. [1] on the sequential use of Sorafenib following Sunitinib failure in metastatic RCC patients should be regarded as an extremely intriguing report.

On the basis of a non-extraordinary time-to-progression (TTP) of just 16 weeks, the authors concluded that “... although well tolerated, Sorafenib shows limited efficacy in Sunitinib-refractory mRCC".

C. Paglino · C. Porta $(\bowtie)$

Medical Oncology, I.R.C.C.S. San Matteo

University Hospital Foundation,

Piazzale C. Golgi 19, 27100 Pavia, Italy

e-mail: c.porta@smatteo.pv.it
In our opinion, these conclusions should be reconsidered in a broader perspective on the light of all the evidence presently available on this hot topic.

Indeed, in the past few years, a number of retrospective studies [2-11], together with a single prospective report [12], and the subgroup analysis of Sorafenib's European Union and United States (US) expanded access programs $[13,14]$, have been published or presented in abstract form, dealing with the sequential use of the two multikinase inhibitors Sunitinib and Sorafenib (Fig. 1).

Even though highly biased by their retrospective nature (and indeed, the prospective nature of the Di Lorenzo's study is the major strength of this study), it is hard to disregard the findings deriving by all these reports, first because they collected more than 550 patients as a whole, and second because there is a constance in the results reported by different studies, performed by different groups, in different parts of the world.

From the analysis of these studies, it is evident that following the failure to one multikinase inhibitors, further PFS/TTP benefit is attainable switching to the other drug; furthermore, all these study seem to suggest a somewhat strange, but intriguing finding, i.e., a longer overall PFS with the Sorafenib-Sunitinib sequence, when compared with the opposite one (Table 1).

In addition, it is this finding (which, once again should presently be taken with great caution) that seems to be even reinforced by the Di Lorenzo study.

The question of using or not multikinase inhibitors in sequence is all, but trivial; indeed, since the pivotal phase III RECORD-1 trial demonstrated not only that the mTOR inhibitor Everolimus was clinically beneficial in patients already treated with multikinase inhibitors, but also that it is as effective after both drugs as after one [15], the question of what should be regarded as the ideal-or at 
Fig. 1 Reports dealing with the sequential use of the two multikinase inhibitors Sunitinib and Sorafenib in advanced kidney cancer patients

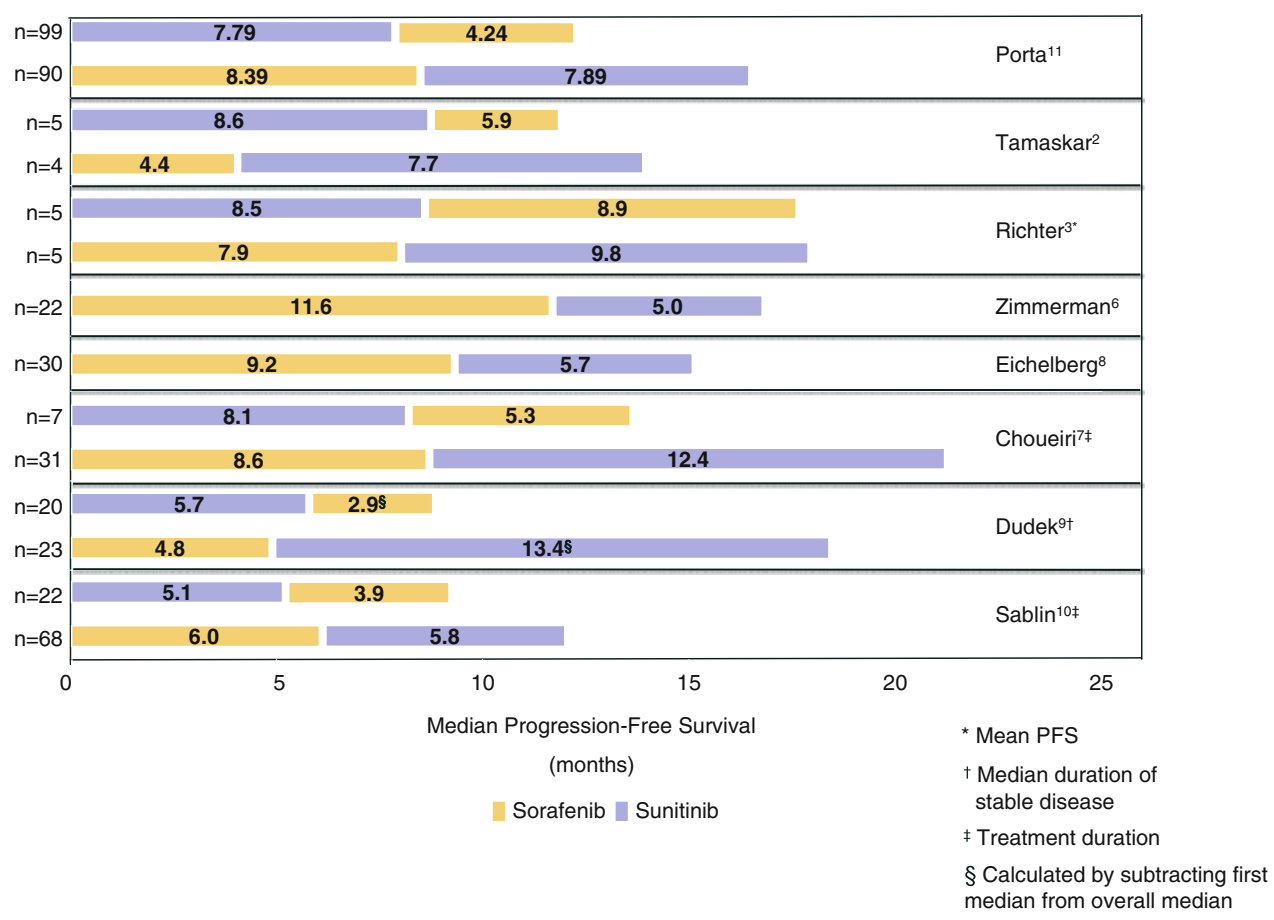

Table 1 Summary of the results of retrospective studies dealing with the sequence Sunitinib $\rightarrow$ Sorafenib or vice versa, i.e., Sorafenib $\rightarrow$ Sunitinib

\begin{tabular}{lcc}
\hline & Number of patients & \\
\cline { 2 - 3 } & Sunitinib $\rightarrow$ Sorafenib & Sorafenib $\rightarrow$ Sunitinib \\
\hline Tamaskar et al. [2] (retrospective) & 5 & 4 \\
Richter et al. [3] (retrospective) & 5 & 5 \\
Mancuso et al. [4] (phase II) & 13 & - \\
US-ARCCS [14] (expanded access) & 13 & - \\
Sepulveda et al. [5] (prospective) & 20 & - \\
Zimmermann et al. [6] (retrospective) & - & 22 \\
Shepard [12] (phase II) & 24 & - \\
Choueiri et al. [7] (retrospective) & 7 & 31 \\
Eichelberg et al. [8] (retrospective) & - & 44 \\
Dudek et al. [9] (retrospective) & 20 & 29 \\
Di Lorenzo (phase II) & 52 & - \\
EU-ARCCS [13] (expanded access) & 69 & - \\
Sablin et al. [10] (retrospective) & 22 & 68 \\
Porta et al. [11] (retrospective) & 99 & 90 \\
Total & 349 & 293 \\
& & \\
\hline
\end{tabular}

least, the best-sequence is becoming more and more important, considering also that very limited treatment options, if any, are presently available for patients who fail an mTOR inhibitor.

Besides, these key clinical considerations, the surprising finding that a lower PFS is observed using Sunitinib after Sorafenib, deserves further insights; is it a problem of toxicity that renders patients treated with the more potent drug Sunitinib less able to receive the other multikinase inhibitor?

Or may we hypothesize that Sunitinib's potency could also be its Achilles' heel?

Indeed, intriguing recent pre-clinical results suggest that "... potent angiogenesis inhibition", such the one exerted by Sunitinib, "can alter the natural history of tumors by increasing invasion and metastasis" [16]. 
A number of presently ongoing trials, e.g. the SWITCH one (Sorafenib/Sunitinib cross-over, NCT00732914), will probably help in solving this dilemma in the near future, but presently we have to deal with the above evidence to make our everyday clinical decisions, and this evidence, in our opinion, support the lack of cross resistance between the two multikinase inhibitors (which, indeed, do not share all the same targets, nor have the same affinity to each target).

\section{Conflict of interest None.}

\section{References}

1. Di Lorenzo G, Cartenì G, Autorino R et al (2009) Phase II study of sorafenib in patients with sunitinib-refractory metastatic renal cell cancer. J Clin Oncol 27:4469-4474

2. Tamaskar I, Garcia JA, Elson P et al (2008) Antitumor effects of sunitinib or sorafenib in patients with metastatic renal cell carcinoma who received prior antiangiogenic therapy. J Urol 179:81-86

3. Richter S, Pfister D, Thur D et al (2009) Second-line treatment of progressive metastatic renal cell cancer with temsirolimus following first-line therapy with sunitinib or sorafenib. Onkologie 31:324 (abstract V684)

4. Mancuso AP, Donato De Paola E, Catalano A et al. (2009) Phase II dose escalation study of sorafenib in patients with metastatic renal cell carcinoma (mRCC) who have had prior treatment with VEGFR-TKI antiangiogenic treatment. J Clin Oncol 27 Suppl (abstract e16027)

5. Sepulveda J, Maroto P, Andres R et al. (2008) Sorafenib as a second-line and sequential therapy for patients with metastatic renal cell carcinoma (mRCC): analysis for safety and activity on sunitinib progressive pts. J Clin Oncol 2008;26 Suppl (abstract 16100)
6. Zimmermann K, Schmittel A, Steiner U et al (2009) Sunitinib treatment for patients with advanced clear-cell renal-cell carcinoma after progression on sorafenib. Oncology 76:350-354

7. Choueiri TK, Brick AJ, McDermott D et al (2008) Treatment and dosing patterns for angiogenesis inhibitor (AIS) therapies in patients with metastatic renal cell carcinoma (mRCC). Ann Oncol 19(Suppl):viii191 (abstract 593P)

8. Eichelberg C, Heuer R, Chun FK et al (2008) Sequential use of the tyrosine kinase inhibitors sorafenib and sunitinib in metastatic renal cell carcinoma: a retrospective outcome analysis. Eur Urol 54:1373-1378

9. Dudek AZ, Zolnierek J, Dham A et al (2009) Sequential therapy with sorafenib and sunitinib in renal cell carcinoma. Cancer 115:61-67

10. Sablin MP, Negrier S, Ravaud A et al (2009) Sequential sorafenib and sunitinib for renal cell carcinoma. J Urol 182:29-34

11. Porta C, Procopio G, Sabbatini R et al (2009) Retrospective analysis of the sequential use of sorafenib and sunitinib in patients with advanced renal cell carcinoma (RCC). Eur Urol (abstract 252)

12. Shepard R, Rini B, Garcia JA et al (2008) A multicenter prospective trial of sorafenib in patients (pts) with metastatic clear cell renal cell carcinoma (mccRCC) refractory to prior sunitinib or bevacizumab. J Clin Oncol 26 Suppl (abstract 5123)

13. Beck J, Bajetta E, Escudier B et al (2007) A large open-label, non-comparative, phase III study of the multi-targeted kinase inhibitor sorafenib in European patients with advanced renal cell carcinoma. Eur J Cancer 5(Suppl):300 (abstract O\#4506)

14. Stadler WM, Figlin RA, McDermott DF et al (2010) Safety and efficacy results of the advanced renal cell carcinoma sorafenib expanded access program in North America. Cancer 2010 (E-pub ahead of print)

15. Motzer RJ, Escudier B, Oudard S et al (2008) Efficacy of everolimus in advanced renal cell carcinoma: a double-blind, randomised, placebo-controlled phase III trial. Lancet 372:449-456

16. Paez-Ribas M, Allen E, Hudock J et al (2009) Antiangiogenic therapy elicits malignant progression of tumors to increased local invasion and distant metastasis. Cancer Cell 15:167-170 